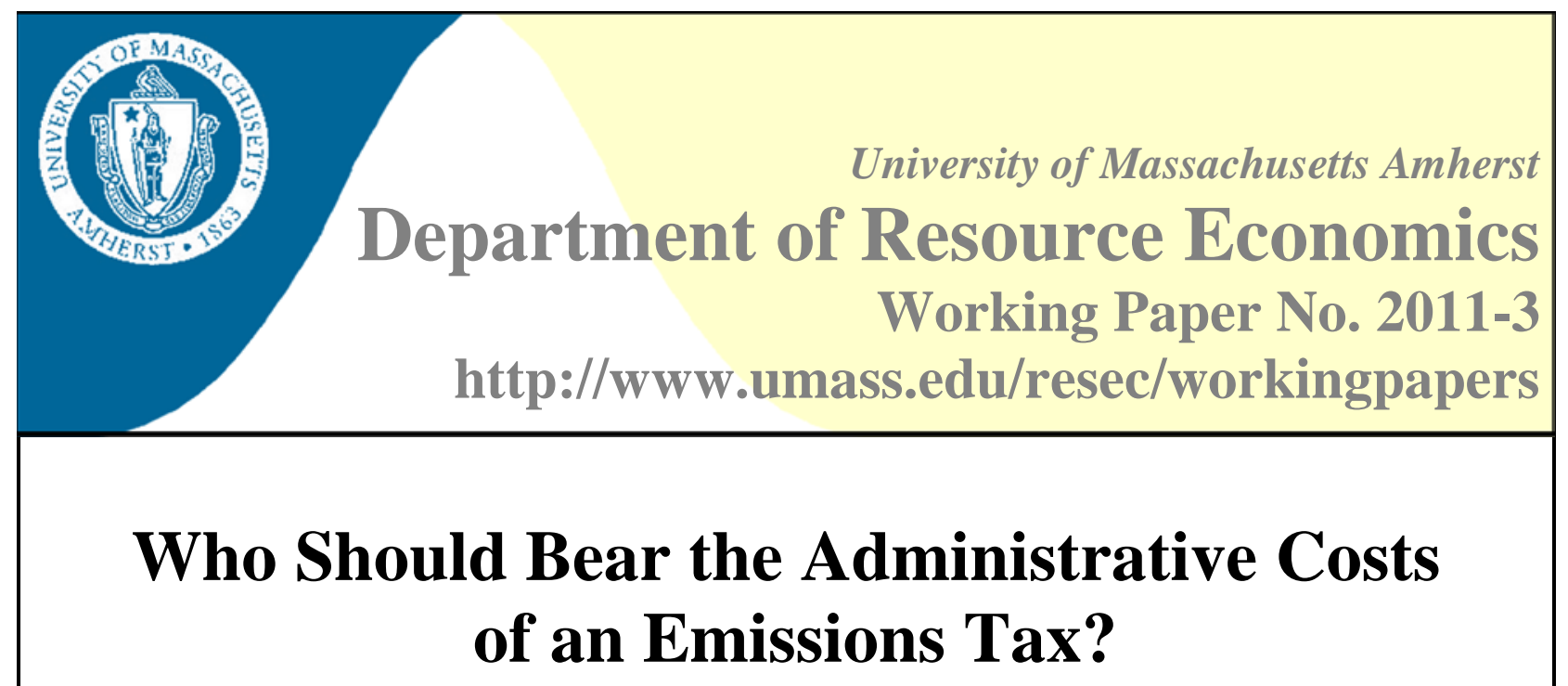

John K. Stranlund ${ }^{1}$ and Carlos A. Chavez ${ }^{2}$

Abstract:

All environmental policies involve administrative costs, the costs of implementing and managing policies that extend beyond abatement costs. We examine theoretically the optimal distribution of these costs between the public and regulated sources of pollution. The distribution of administrative costs affects social welfare only if public funds are more expensive than private funds, or if the distribution of administrative costs affects the size of a regulated industry. If having the public take on a larger part of administrative costs increases the size of the industry and this does not lead to lower emissions for a given emissions tax, then it is optimal to make the pollution sources bear all of the administrative costs. A necessary, but not sufficient, reason for having the public bear part of the cost burden is if aggregate emissions decrease as a result.

Keywords: Emissions Taxes, Pigouvian Taxes, Administrative Costs, Pollution Control

JEL Classification: C92, H41

\footnotetext{
${ }^{1}$ John K. Stranlund, Department of Resource Economics

University of Massachusetts, 222 Stockbridge Hall

80 Campus Center Way, Amherst, MA 01003-9246

E: stranlund@resecon.umass.edu P: 413-545-6328 F: 413-545-5853

${ }^{2}$ Carlos A. Chavez, Departamento de Economia

Facultad de Ciencias Economicas y Administrativas

Universidad de Concepcion

Casilla (Box) 1987, Concepcion, Chile

E: cchavez@udec.cl P: 56-41-2203067 F: 56-41-2254591
} 
March 2011

\title{
Who should bear the administrative costs of an emissions tax?
}

\author{
JOHN K. STRANLUND \\ Department of Resource Economics \\ University of Massachusetts-Amherst \\ CARLOS A. CHÁVEZ \\ Departamento de Economía \\ Universidad de Concepción
}

\begin{abstract}
All environmental policies involve administrative costs, the costs of implementing and managing policies that extend beyond abatement costs. We examine theoretically the optimal distribution of these costs between the public and regulated sources of pollution. The distribution of administrative costs affects social welfare only if public funds are more expensive than private funds, or if the distribution of administrative costs affects the size of a regulated industry. If having the public take on a larger part of administrative costs increases the size of the industry and this does not lead to lower emissions for a given emissions tax, then it is optimal to make the pollution sources bear all of the administrative costs. A necessary, but not sufficient, reason for having the public bear part of the cost burden is if aggregate emissions decrease as a result.

Keywords: Emissions Taxes, Pigouvian Taxes, Administrative Costs, Pollution Control JEL Codes: L51, Q58

Correspondence to: John K. Stranlund, Department of Resource Economics, 222 Stockbridge Hall, 80 Campus Center Way, University of Massachusetts-Amherst, Amherst, MA 01003, USA. Phone: (413) 545-6328, Fax: (413) 545-5853, E-mail: stranlund@ resecon.umass.edu.
\end{abstract}




\section{Who should bear the administrative costs of an emissions tax?}

\section{Introduction}

Any environmental policy involves costs beyond what would normally be categorized as abatement costs. These costs include the costs of monitoring polluters for compliance and imposing sanctions when a violation is found. In addition, resources are expended for monitoring measures of policy performance, conducting research, and record keeping and reporting by both regulated firms and regulators. For environmental taxes, these costs include the costs of setting up and maintaining a system for collecting tax revenue. Perhaps for lack of a better term, these costs may be referred to as administrative costs. These are the costs of implementing and managing environmental policies that extend beyond abatement costs, and they are typically borne by both regulated firms and regulators.

Policy analysts have long known that administrative costs can affect the setting of optimal emissions taxes. Polinksy and Shavell (1982) show how fixed and variable administrative costs affect the level of a uniform Pigouvian tax, while Cremer and Gahvari (2002) and Stranlund, Chavez, and Villena (2009) show that enforcement costs can produce discriminatory emissions taxes. Researchers also understand that administrative costs can affect the relative efficiency of different kinds of environmental taxes. For example, Smulders and Vollebergh (2001) examine how administrative costs, particularly monitoring costs, determine the use of emissions and input taxes with specific reference to taxing $\mathrm{CO} 2$ emissions or taxing energy products. Schmutzler and Goulder (1997) provide another analysis of mixed environmental taxes that is motivated by differences in monitoring costs. ${ }^{1}$

\footnotetext{
${ }^{1}$ Others have investigated how administrative costs can affect the relative efficiency of taxes and other policy instruments. For example, Kampas and White (2004) provide an empirical analysis of the relative efficiency of several different policies for the control of an agricultural nonpoint
} 
While it is well known that administrative costs can be important determinants of the design and performance of environmental policies, in the literature on emissions taxes the distribution of administrative costs between the public (via government regulators) and polluting firms is taken for granted. No one to our knowledge has addressed the question of who should bear the administrative costs associated with emissions taxes, the public, regulated pollution sources, or some combination. ${ }^{2}$ In this paper we seek to provide this missing element.

Policymakers cannot sidestep the problem of distributing administrative costs and have confronted it in a variety of ways. Many emissions control policies require that regulated firms bear the costs of monitoring and reporting their own emissions while the government takes on the burden of checking emissions reports and sanctioning violations. (The SO2 Allowance Trading Program is a good example). Some programs collect administrative fees from pollution sources to help finance enforcement and other activities. For example, Title V of the 1990 amendments to the U.S. Clean Air Act requires most large sources and some smaller sources of air pollution to obtain operating permits, and to pay an annual fee per ton of each regulated pollutant to cover the costs of administering the permit program. (See http://www.epa.gov/air/oaqps/permits/, last accessed February 27, 2011). We know of no study that addresses whether it is efficient to collect such fees.

Our study gives us insight into questions like this in the case of emissions taxes. We identify two avenues through which the distribution of administrative costs between regulated

pollutant. They find that the presence of administrative costs favors an input tax even though this instrument does not minimize aggregate abatement costs.

${ }^{2}$ This is largely true of other pollution control instruments as well, although some attention has been devoted to the subject in fisheries management where there may be a greater expectation that regulated entities should bear administrative costs. See Tietenberg (2003) and Schrank, Arnason, and Hannesson (2003) for examples of fees on fishing quota owners for recovering public management costs in several fisheries. 
pollution sources and the general public can affect social welfare. If public funds are more expensive than private funds due to the deadweight costs of taxes used to generate government revenue, then there is an incentive to shift the burden of administrative costs onto regulated firms. The other way in which the distribution of administrative costs can affect social welfare is if it impacts the size of the regulated industry. Shifting administrative costs from regulated firms to the public can increase their number because production becomes more profitable.

When public funds and private funds are equally costly and the burden of administrative costs does not affect the number of regulated firms, the distribution of these costs does not affect the social costs and benefits of controlling emissions with an emissions tax. Consequently, any distribution is efficient as long as it is accompanied by an optimal tax. However, the chosen distribution will affect the level of an optimal tax. In fact, the results of Polinsky and Shavell (1982) suggest that the optimal emissions tax can be less than, greater than, or equal to marginal damage depending on the exogenous distribution of administrative costs and the relative importance of fixed versus variable components of these costs. These results are relevant when there is not an efficiency consequence of choosing a particular distribution of administrative costs, or when these consequences exist but are not accounted for when specifying the distribution in the regulation.

Our contribution is in showing when the distribution of administrative costs matters and specifying the optimal distribution when it does. In particular, when industry size is not fixed, the ultimate effect of administrative costs on aggregate emissions is a key element of the optimal distribution of these costs. If the number of regulated firms increases with a shift in administrative costs toward the general public, two countervailing effects determine how aggregate emissions change. For a given emissions tax and fixed price of the industry's output, 
more firms in the industry increases aggregate emissions. On the other hand, more firms decrease the price of output, which, in turn, reduces emissions of individual firms because they reduce output. If the former effect dominates, or if the effects cancel each other out, so that having the public bear a greater part of administrative costs does not decrease aggregate emissions, it is optimal to have the firms bear all administrative costs. However, a necessary, but not sufficient, condition for it to be optimal for the public to bear part of the administrative costs of an emissions tax is that removing some of the administrative cost burden from firms reduces aggregate emissions.

Finally, while Polinsky and Shavell (1982) argued that a Pigouvian tax can be greater than, less than, or equal to marginal damage when the distribution of administrative costs is exogenous, we show that when the distribution of administrative costs impacts social welfare and is chosen optimally, the accompanying emissions tax should never exceed marginal damage.

The rest of the paper proceeds as follows. In the next section we lay out the elements of our model and determine how the policy parameters, an emissions tax and distribution of administrative costs, affect equilibrium market and emissions outcomes. Section 3 contains the main results of our analysis, those concerning the optimal distribution of administrative costs. We conclude in section 4.

\section{A model of an emissions tax with administrative costs}

The regulatory model of this paper is standard: a regulator commits to the parameters of an emissions tax policy and communicates these to the regulated firms before they make their choices. In this section we present a model of the distribution of administrative costs under an 
emissions tax and the effects of these policy choices on equilibrium output, emissions, and industry size.

\subsection{Basic elements}

Throughout we consider competitive firms that produce an output $q$ at price $p$. All the firms in our model belong to the same industry, but we make this assumption only to ease the exposition. Firms produce emissions $x$ as a byproduct of production. Emissions are uniformly mixed and cause damage at constant rate $d .{ }^{3} \mathrm{~A}$ firm's cost function is $c(q, x, \lambda)$, which is strictly increasing in output, decreasing in emissions, and strictly convex in $q$ and $x$. Moreover, we make the common assumption that $c_{q x}<0$ so that firms' marginal production costs are decreasing in their emissions. $\lambda$ is a parameter that is used to order the firms: we will refer to $\lambda$ as a firm's type. The firms' emissions are controlled with a per unit tax $t$. We assume throughout the paper that enforcement is sufficient to induce full compliance, which means that each operating firm submits a truthful report of its tax liability to the authorities. ${ }^{4}$

As noted in the introduction, the administrative costs associated with maintaining any environmental policy are likely to come from several sources (e.g., monitoring and enforcement, research, record keeping, etc). Rather than model each of these sources, we aggregate all such costs and make simple assumptions about how they vary. First, we assume that aggregate administrative costs are strictly increasing in the stringency of an emissions control policy, given

\footnotetext{
${ }^{3}$ We assume constant marginal damage to simplify the analysis. This assumption does not change our results.

${ }^{4}$ Recent work by Stranlund, Chavez, and Villena (2009) suggests that there is a very limited set of circumstances under which it may be efficient to implement emissions taxes that are not fully enforced. Moreover, designing an enforcement strategy that induces full compliance is very simple, because it only requires that firms face an expected marginal penalty that exceeds the tax.
} 
a fixed number of regulated firms. This implies that administrative costs are strictly increasing in an emissions tax. This assumption is justified largely by the monitoring and enforcement literature, which suggests that firms have a stronger incentive to evade a higher emissions tax. ${ }^{5}$ Following Polinsky and Shavell (1982), we may refer to the administrative costs that vary with the stringency of environmental control as variable administrative costs. ${ }^{6}$

However, there are administrative costs that do not change with the stringency of the regulation but may vary from firm to firm and, in aggregate, increase with the number of regulated firms, holding the policy instrument (i.e., an emissions tax) constant. For example, suppose that each firm is required to install a continuous emissions monitoring device (as in the SO2 Allowance Trading program and other emissions trading programs). The costs of installing and operating this technology for a particular plant may not change with the stringency of the regulation, because the device has to be installed if the plant is to operate and because it has to run non-stop. However, these costs may vary from firm to firm, perhaps because some firms have to install multiple devices if they have multiple stacks to monitor. In addition, more regulated firms require the installation and operation of more devices, which adds to aggregate

\footnotetext{
${ }^{5}$ The theoretical literature on the enforcement of emissions taxes includes Harford (1978 and 1987), Sandmo (2002), Montero (2002), Cremer and Gahvari (2002), Macho-Stadler and PerezCastrillo (2006), and Stranlund, Chávez, and Villena (2009). All note that firms have a greater incentive to evade higher taxes. Therefore, regulators must expend greater enforcement resources to maintain compliance with higher emissions taxes.

${ }^{6}$ Our model borrows much from Polinsky and Shavell (1982). The main difference between our effort and theirs is that we look for the optimal distribution of administrative costs while they treat it as exogenous. There are some less critical but nevertheless important differences between the models. We allow for a difference in the costs of public versus private funds and we incorporate an output market into the analysis. Polinsky and Shavell do not include either of these features. They also assume that variable administrative costs increase with the total tax liability of individual firms. (This is assumed by Brock and Evans (1985) as well). We think this assumption is less plausible than our assumption that variable administrative costs increase with the tax rate, because the former assumption allows administrate costs to fall with more stringent environmental regulation.
} 
costs. Plant characteristics like location, abatement and production technologies may also generate differences in monitoring costs among firms. We will refer to costs that may differ across firms but do not change with the stringency of the regulation as fixed administrative costs. We do not include administrative costs that are fixed with respect to both the stringency of a regulation and the number of regulated firms. This is a simplifying assumption that does not affect our results as long as these costs are not so high that any level of regulation is inefficient.

Mainly as a device to simplify the analysis of the optimal distribution of the burden of administrative costs, we assume that all administrative costs are apportioned to individual firms that operate under an emissions tax. This does not mean that the firms will bear the costs apportioned to them; it does, however, allow us to describe aggregate administrative costs as an aggregation of these costs over regulated firms.

Under our assumptions thus far, let $m(t, \lambda)$ denote the administrative cost that are apportioned to an operating $\lambda$-type firm. These functions includes both variable and fixed components so $m(t, \lambda)>0$ and $m_{t}(t, \lambda)>0$ for all $t \geq 0$.

Let $\alpha$ be the share of the administrative costs apportioned to a firm that is borne by the government- $-(1-\alpha)$ is the share that is borne by the firm — and assume for simplicity that this share is constant across firms. The efficient level of $\alpha$ is the main question addressed in this paper. There is one final component of administrative costs. Typically, governments must raise revenue with distortionary taxes, which implies that a dollar of government expenditure costs more than a dollar. Consequently, let the marginal cost of public funds be the constant $\mu \geq 1$, so 
the cost of one dollar of funds to finance administrative costs is $\alpha \mu+1-\alpha=1+\alpha(\mu-1)$.

Clearly this term is increasing in the marginal cost of public funds. ${ }^{7}$

Given $\alpha$ and $t$ the administrative costs borne by an operating $\lambda$-type firm are $(1-\alpha) m(t, \lambda)$, while the government takes on $\alpha \mu m(t, \lambda)$. Implementation of this distribution is done with fees and subsidies that we do not model explicitly. Suppose for the sake of an illustration that the only administrative costs are monitoring costs. The default distribution of these costs has the firm paying for installing and operating an emissions monitoring technology and submitting emissions reports to the government, while the government bears the costs of monitoring the firm's reports and data related to the operation of the monitoring device. If it turns out to be optimal that firms shoulder all monitoring costs, then the government charges the firm a fee to recover the costs of its activities. If it is optimal that the public bear all administrative costs, then a subsidy is paid to the firm to cover the costs of its monitoring and reporting system. Of course, sharing administrative costs is possible, and fees and subsides can be structured to implement any sharing requirement. It is important that fees charged to firms to recover public administrative costs are in addition to their emissions tax payments. Moreover, fees and subsidies are levied in a way that does not distort their output and emissions decisions.

For easy reference, Table 1 provides the list of the most important variables and functions used in this paper.

\footnotetext{
${ }^{7}$ Some may wonder why we model the difference between public and private costs when the government could use emissions tax receipts to finance its share of administrative costs. Using the tax revenue in this way would still involve an opportunity cost if that revenue could be used to help finance other government activities that would instead have been financed with other distortionary taxes.
} 
Table 1: Key notation

\begin{tabular}{|c|l||}
\hline Symbol & Description \\
\hline$q, Q$ & A firm's output and aggregate output: $\hat{q}$ and $\hat{Q}$ are equilibrium values. \\
\hline$p$ & Output price: $\hat{p}$ is equilibrium price. \\
\hline$x, X$ & A firm's emissions and aggregate emissions: $\hat{x}$ and $\hat{X}$ are equilibrium values. \\
\hline$d$ & Constant marginal damage. \\
\hline$\lambda$ & Firm type, used to order firms. \\
\hline$\lambda^{m}, \hat{x}^{m}$ & Type of the least profitable firm in the industry: $\hat{\lambda}^{m}$ is the equilibrium value. \\
\hline$c(q, x, \lambda)$ & Autput and emission of the least profitable firm in equilibrium. \\
\hline$t$ & Emissions tax. \\
\hline$m(t, \lambda)$ & Administrative cost apportioned to an operating $\lambda$-type firm. \\
\hline$\alpha$ & Proportion of administrative costs borne by the government. \\
\hline$\mu$ & Constant marginal cost of public funds. \\
\hline$\pi$ & A firm's profit. \\
\hline
\end{tabular}

\subsection{Firms' choices}

Given $t, \alpha$, and enforcement sufficient to motivate each firm's full compliance, a $\lambda$-type firm that continues to operate under the emissions tax chooses emissions and output to maximize profit:

$$
\pi=p q-c(q, x, \lambda)-t x-(1-\alpha) m(t, \lambda) .
$$

We restrict our attention to policies that induce interior choices of output and emissions for all operating firms. The first order conditions for a firm's output and emissions are:

$$
p-c_{q}(q, x, \lambda)=0 \text { and }-c_{x}(q, x, \lambda)-t=0
$$

respectively. As is standard, competitive firms choose their output to equate marginal production costs to the output price, and they choose emissions to equate their marginal abatement costs to the emissions tax. These conditions implicitly define the firm's choices of production and emissions as: 


$$
q(t, p, \lambda) \text { and } x(t, p, \lambda)
$$

Let $S=c_{q q} c_{x x}-\left(c_{q x}\right)^{2}$ denote the determinant of the Hessian matrix of the cost function. $S>0$ is required for concavity of the firms' profit functions. In the usual manner, find the following comparative statics:

$$
q_{p}=c_{x x} / S>0, q_{t}=c_{q x} / S<0, x_{p}=-c_{q x} / S>0 \text {, and } x_{t}=-c_{q q} / S<0 .
$$

These comparative statics indicate that a firm's emissions and output are both increasing in the product price, and both are decreasing in the emissions tax.

Now let us turn to the entry and exit condition that determines the size of the industry. Using (1) and (3), define the maximum profit for a $\lambda$-type firm as:

$$
\pi(t, \alpha, p, \lambda)=p q(t, p, \lambda)-c(q(t, p, \lambda), x(t, p, \lambda), \lambda)-t x(t, p, \lambda)-(1-\alpha) m(t, \lambda) .
$$

Assume that firms are continuously ordered so that $\pi_{\lambda}(t, \alpha, p, \lambda)>0$ for all $t$ and for all $\alpha \in[0,1] .{ }^{8}$ Suppose that $\lambda$ is then distributed according to the density function $f(\lambda)$ on support $[0, \bar{\lambda}]$, with $\pi(t, \alpha, p, \lambda=0)<0$ and $\pi(t, \alpha, p, \bar{\lambda})>0$. These imply, for given policy parameters $t$ and $\alpha$, that there is a unique cut-off value of $\lambda>0$ below which firms earn negative profit, and thus will not operate. Firms identified by $\lambda$ 's greater than or equal to the cutoff $\lambda$ continue to operate. The cut-off value of $\lambda$ is determined as the implicit solution to $\pi(t, \alpha, p, \lambda)=0$. Let this value be

$$
\lambda^{m}=\lambda^{m}(t, \alpha, p)
$$

Note that a higher (lower) value of $\lambda^{m}$ indicates that the industry is smaller (larger). Substitute

(6) into (5) to obtain $\pi\left(t, \alpha, p, \lambda^{m}\right) \equiv 0$, and

\footnotetext{
${ }^{8}$ The ordering of firms requires $-c_{\lambda}-(1-\alpha) m_{\lambda}>0$; that is, firms' production costs plus their share of their own administrative costs decrease with $\lambda$.
} 


$$
\lambda_{t}^{m}=\left(x+(1-\alpha) m_{t}\right) / \pi_{\lambda}>0, \lambda_{\alpha}^{m}=-m / \pi_{\lambda}<0 \text {, and } \lambda_{p}^{m}=-q / \pi_{\lambda}<0 .
$$

These relations indicate that the regulation reduces the number of active firms as the tax increases or if more of the administrative cost burden is placed on the firms, holding the output price constant. However, the number of active firms increases with a higher output price. We recognize that some of these effects may be zero empirically. In particular, the firms'

administrative cost burden may have a negligible effect on the number of firms if these costs are a very small part of their total costs. Since our main results depend on whether the tax and administrative cost burden change the number of firms in an industry, we allow $\lambda_{t}^{m}, \lambda_{\alpha}^{m}$, and $\lambda_{p}^{m}$ to be negligible

\subsection{Market equilibrium}

We now specify the output market equilibrium in terms of the policy parameters $t$ and $\alpha$. Begin with the supply function:

$$
Q^{S}(t, \alpha, p)=\int_{\lambda^{m}}^{\bar{\lambda}} q(t, p, \lambda) f(\lambda) d \lambda
$$

Define

$$
q^{m}=q\left(t, p, \lambda^{m}\right) \text { and } x^{m}=x\left(t, p, \lambda^{m}\right),
$$

as the output and emissions of the cut-off firm in the industry. Then:

$$
\begin{aligned}
& Q_{p}^{S}=\int_{\lambda^{m}}^{\bar{\lambda}} q_{p} f(\lambda) d \lambda-\lambda_{p}^{m} q^{m} f\left(\lambda^{m}\right)>0 \\
& Q_{t}^{S}=\int_{\lambda^{m}}^{\bar{\lambda}} q_{t} f(\lambda) d \lambda-\lambda_{t}^{m} q^{m} f\left(\lambda^{m}\right)<0 \\
& Q_{\alpha}^{S}=-\lambda_{\alpha}^{m} q^{m} f\left(\lambda^{m}\right)>0
\end{aligned}
$$


As expected the industry supply function is increasing in the price of output. Note that the first term of (10) captures the price effect given a fixed number of firms, while the second term is an industry size effect that captures the increase in quantity supplied because an increase in output price may attract more firms to the industry. The industry supply function is decreasing in the emissions tax because each operating firm's output and the size of the industry are decreasing in the tax. Note that the effect of $\alpha$ on the supply function is only an industry-size effect. Increasing the public's share of administrative costs increases industry supply if it increases the number of firms in the industry. If $\lambda_{\alpha}^{m} \approx 0$ then $Q_{\alpha}^{S} \approx 0$.

Let $Q^{D}(p)$ be the demand function for the industry's output, and assume that it is downward sloping. Then the equilibrium price is the solution to $Q^{S}(t, \alpha, p)=Q^{D}(p)$. Denote this price as $\hat{p}$, and note that it is a function of the policy parameters $t$ and $\alpha$. All equilibrium values from here on will be indicated with a "hat." From the market-clearing condition obtain:

$$
\hat{p}_{t}=-Q_{t}^{S} /\left(Q_{p}^{S}-Q_{p}^{D}\right)>0 \text { and } \hat{p}_{a}=-Q_{\alpha}^{S} /\left(Q_{p}^{S}-Q_{p}^{D}\right) \leq 0
$$

The signs of $\hat{p}_{t}$ and $\hat{p}_{\alpha}$ follow from $Q_{p}^{S}-Q_{p}^{D}>0,(11)$, and (12), and indicate that the equilibrium price of the industry's output is increasing in the emissions tax and non-increasing in the share of administrative costs borne by the public. Substitute (12) into (13) to obtain

$$
\hat{p}_{a}=\lambda_{\alpha}^{m} q^{m} f\left(\lambda^{m}\right) /\left(Q_{p}^{S}-Q_{p}^{D}\right)
$$

and note that $\hat{p}_{\alpha} \approx 0$ if $\lambda_{\alpha}^{m} \approx 0$. Thus, given a downward sloping demand curve, a change in the distribution of administrative costs changes the equilibrium output price if and only if it changes the size of the industry.

We will also consider the optimal distribution of enforcement costs when demand for the industry's output is perfectly elastic. This case is important because it is relevant for domestic 
environmental control of firms that are a relatively small part of an international market. In this case, a change in the distribution of administrative costs can change the size of the industry without changing the equilibrium permit price.

With the equilibrium product price we can use (3) and (6) to define the equilibrium values of $q$ and $x$ for operating firms, as well as the cut-off firm type:

$$
\hat{q}=q(t, \hat{p}, \lambda), \hat{x}=x(t, \hat{p}, \lambda), \text { and } \hat{\lambda}^{m}=\lambda^{m}(t, \alpha, \hat{p})
$$

We will see that the effects of the policy parameters on firms' equilibrium levels of output have no bearing on the optimal choices of the tax and distribution of administrative costs. The effects of the policy parameters on their choices of emissions and the size of the industry are what matter. The effects of the tax are indeterminate at this level of generality because they depend on direct effects and countervailing indirect price effects. For example, the effect of the tax on individual emissions is $\hat{x}_{t}=x_{t}+x_{p} \hat{p}_{t}$. The direct effect of the emissions tax is to reduce operating firms' emissions $\left(x_{t}<0\right)$, but an increase in the tax can increase the output price, which has a positive indirect effect on an individual firm's emissions $\left(x_{p} \hat{p}_{t}>0\right)$. Despite this indeterminacy, we will assume that equilibrium firm-level emissions are decreasing in the tax:

$$
\hat{x}_{t}=x_{t}+x_{p} \hat{p}_{t}<0
$$

The effect of the tax on the equilibrium industry size is similarly indeterminate. However, we assume

$$
\hat{\lambda}_{t}^{m}=\lambda_{t}^{m}+\lambda_{p}^{m} \hat{p}_{t} \geq 0
$$

because it seems unlikely that increasing firms' costs (with an increased tax) can increase their profitability so that more firms enter the industry. 
The effect of the public's share of administrative costs on individual firms' emissions is not ambiguous. This effect is

$$
\hat{x}_{\alpha}=x_{p} \hat{p}_{\alpha} \leq 0
$$

which is non-increasing because $x_{p}>0$ and $\hat{p}_{\alpha} \leq 0$. The effect of $\alpha$ on the equilibrium size of the industry is also unambiguous, but it takes some effort to show this. Note first that

$$
\hat{\lambda}_{\alpha}^{m}=\lambda_{\alpha}^{m}+\lambda_{p}^{m} \hat{p}_{\alpha}
$$

Suppose that the industry faces a downward sloping demand curve. Substitute (14) into (19) and collect terms to obtain

$$
\hat{\lambda}_{\alpha}^{m}=\lambda_{\alpha}^{m}\left(\frac{\left(Q_{p}^{S}-Q_{p}^{D}\right)+\lambda_{p}^{m} q^{m} f\left(\lambda^{m}\right)}{\left(Q_{p}^{S}-Q_{p}^{D}\right)}\right) .
$$

Substitute (10) in for $Q_{p}^{S}$ in the numerator of (20) and simplify to

$$
\hat{\lambda}_{\alpha}^{m}=\lambda_{\alpha}^{m} K \leq 0 \text {, where } K=\left(\int_{\lambda^{m}}^{\bar{\lambda}} q_{p} f(\lambda) d \lambda-Q_{p}^{D}\right) /\left(Q_{p}^{S}-Q_{p}^{D}\right)>0
$$

$K$ is strictly positive because $q_{p}>0, Q_{p}^{D}<0$, and $Q_{p}^{S}-Q_{p}^{D}>0$. Therefore, the sign of $\hat{\lambda}_{\alpha}^{m}$ is equal to the sign of $\lambda_{\alpha}^{m}$, which recall is less than zero, or perhaps negligible.

Our next step is to specify aggregate equilibrium output and emissions. These are

$$
\hat{Q}=\int_{\hat{\lambda}^{m}}^{\bar{\lambda}} \hat{q} f(\lambda) d \lambda
$$

and

$$
\hat{X}=\int_{\hat{\lambda}^{m}}^{\bar{\lambda}} \hat{x} f(\lambda) d \lambda
$$


respectively. Again, we will see that the impacts of the policy variables on equilibrium output do not affect the optimal policy. However, as one would expect, the effects of the policy variables on aggregate emissions have a major impact on their optimal values. These effects are:

$$
\hat{X}_{t}=\int_{\hat{\lambda}^{m}}^{\bar{\lambda}} \hat{x}_{t} f(\lambda) d \lambda-\hat{\lambda}_{t}^{m} f\left(\hat{\lambda}^{m}\right) \hat{x}^{m}<0 .
$$

and

$$
\hat{X}_{\alpha}=\int_{\hat{\lambda}^{m}}^{\bar{\lambda}} \hat{x}_{\alpha} f(\lambda) d \lambda-\hat{\lambda}_{\alpha}^{m} f\left(\hat{\lambda}^{m}\right) \hat{x}^{m},
$$

where $\hat{x}^{m}=x\left(t, \lambda^{m}, \hat{p}\right)$ is the equilibrium emissions of the cut-off firm. While it is not possible at this level of generality to give a definite sign to $\hat{X}_{t}$, as noted above we insist that it must be negative to make an emissions tax worthwhile.

It is not as easy to justify a sign for $\hat{X}_{\alpha}$, but we will see that it plays a critical role in the determination of the optimal distribution of administrative costs. From (14), (18), and (20), we first notice that $\hat{X}_{\alpha}$ is negligible in the important case that $\lambda_{\alpha}^{m}$ is negligible. When $\lambda_{\alpha}^{m}<0$ so that increasing $\alpha$ increases industry size for a given tax and output price, $\hat{X}_{\alpha}$ consists of two effects that work in opposite directions. Holding the output price constant, more firms imply higher aggregate emissions. This effect is captured by $-\hat{\lambda}_{\alpha}^{m} f\left(\hat{\lambda}^{m}\right) \hat{x}^{m}>0$ in (25). However, as the industry expands, the output price falls leading to reduced emissions by each firm in the industry. This is the first term on the right side of (25). If the former industry-size effect dominates then $\hat{X}_{\alpha}>0$, but if the latter price effect dominates then $\hat{X}_{\alpha}<0$. An important special case occurs when the industry faces a perfectly elastic demand curve. When $\lambda_{\alpha}^{m}<0$ and $\hat{p}_{\alpha}=0,(18),(19)$, 
and (25), imply $\hat{X}_{\alpha}=-\lambda_{\alpha}^{m} f\left(\hat{\lambda}^{m}\right) \hat{x}^{m}>0$, indicating that aggregate emissions increase as the public takes on a greater share of administrative costs.

\section{Efficiency: The optimal emissions tax and distribution of administrative costs}

Having specified firm and market reactions to an emissions tax and distribution of administrative costs, we are now ready to characterize their optimal values. The optimal policy will maximize consumer plus producer surplus, less emissions damage and aggregate administrative costs, subject to the equilibrium output and emissions induced by the policy. The social welfare function in terms of the policy variables is:

$$
\begin{aligned}
W(t, \alpha)= & \int_{0}^{\hat{Q}} p(Q) d Q-\int_{\hat{\lambda}^{m}}^{\bar{\lambda}} c(\hat{q}, \hat{x}, \lambda) f(\lambda) d \lambda-d \int_{\hat{\lambda}^{m}}^{\bar{\lambda}} \hat{x} f(\lambda) d \lambda \\
& -(1+\alpha(\mu-1)) \int_{\hat{\lambda}^{m}}^{\bar{\lambda}} m(t, \lambda) f(\lambda) d \lambda .
\end{aligned}
$$

The first term in (26) is consumer surplus: $p(Q)$ is the inverse demand curve for the industry's output. The second and third terms are equilibrium industry costs and pollution damage, and the fourth term is equilibrium administrative costs, including the costs of public funds devoted to administering the emissions tax.

The optimal policy is $(t, \alpha)$ that maximizes $W(t, \alpha)$ subject to $\alpha \in[0,1]$ and $t>0$. Assume that the first-order conditions for this problem are necessary and sufficient to identify an optimal policy. In the Appendix we show that these first-order conditions can be written as:

$$
\begin{aligned}
& W_{t}(t, \alpha)=(t-d) \hat{X}_{t}-(1+\alpha(\mu-1)) \int_{\hat{\lambda}^{m}}^{\bar{\lambda}} m_{t} f(\lambda) d \lambda+\hat{\lambda}_{t}^{m} f\left(\hat{\lambda}^{m}\right) \alpha \mu m\left(t, \hat{\lambda}^{m}\right)=0 \\
& W_{\alpha}(t, \alpha)=(t-d) \hat{X}_{\alpha}-(\mu-1) \int_{\hat{\lambda}^{m}}^{\bar{\lambda}} m f(\lambda) d \lambda
\end{aligned}
$$




$$
+\hat{\lambda}_{\alpha}^{m} f\left(\hat{\lambda}^{m}\right) \alpha \mu m\left(t, \hat{\lambda}^{m}\right)\left\{\begin{array}{l}
\leq 0, \text { if }<0, \text { then } \alpha=0 \\
\geq 0, \text { if }>0, \text { then } \alpha=1 .
\end{array}\right.
$$

The first term of each first order condition captures how the policy variable changes the difference between aggregate abatement cost and pollution damage as aggregate emissions change. (Note that $t$ is equal to aggregate marginal abatement cost). For the emissions tax the sign of this effect depends on whether the optimal tax is greater than, equal to, or less than marginal damage. The effect of changing the public's share of administrative costs also depends on how aggregate emissions change, which is indeterminate in general. All the rest of the terms in the first order conditions involve administrative costs; hence, the absence of these costs results in the standard prescription to set the emissions tax equal to marginal damage.

The effect of the tax on aggregate administrative costs for a fixed number of firms shows up as the second terms in (27) and (28). The last terms of the first order conditions capture how aggregate administrative costs change as the policy variables affect the size of the regulated industry. Take the tax, for example. If increasing this tax decreases the size of the industry, then the higher tax has an indirect benefit in that administrative resources are devoted to fewer firms. On the other hand, having the public take on a greater share of administrative costs can increase aggregate administrative costs if this increases the number of active firms. ${ }^{9}$ The presence of this effect tends to motivate a lower tax, and consequently, less stringent emissions control.

\footnotetext{
${ }^{9}$ Note that the effects of the policy variables on equilibrium industry output (i.e., $\hat{Q}_{t}$ and $\hat{Q}_{\alpha}$ ) do not play a role in the determination of the optimal policy. This is due to the assumption of perfect competition in the output market, because firms choose efficient levels of output, given the policy choice Certainly, the effects of the policy variables on industry output would affect the optimal policy under imperfect competition, because the emissions control policy would be forced to deal with the output market inefficiency Allowing for imperfect competition would be a worthwhile extension of this work.
} 
We are now ready to present the main results of the study, which are contained in several propositions. Our first proposition specifies the avenues through which the distribution of administrative costs has efficiency consequences. All propositions in this paper are proved in the Appendix.

Proposition 1: If $\lambda_{\alpha}^{m} \approx 0$ and $\mu=1$, then, given the choice of an optimal emissions tax, the distribution of administrative costs has no impact on social welfare.

Not surprisingly, one of ways that the distribution of enforcement costs impacts social welfare is through a difference in the costs of public and private funds. The other is not as obvious. The distribution of administrative costs affects social welfare if it affects the size of the regulated industry. Hence, with respect to efficiency we only need to worry about setting the correct distribution of administrative costs if it affects the size of the industry or if public funds devoted to administration are more costly than private funds.

That is not to say that we can ignore the distribution of administrative costs in setting the optimal emissions tax. Even if there is not a unique optimal distribution of these costs, one must be chosen and it is clear in (27) that it affects the level of the tax. For example, suppose that firms are made to bear all administrative costs $(\alpha=0)$. With $\mu=1$, (27) can be written as

$$
t=d+\int_{\hat{\lambda}^{m}}^{\bar{\lambda}} m_{t} f(\lambda) d \lambda / \hat{X}_{t},
$$

which implies that $t<d$ as long as $m_{t}>0$. On the other hand, suppose that the government takes on all administrative costs. Then (27) can be written as 


$$
t=d+\left(\int_{\hat{\lambda}^{m}}^{\bar{\lambda}} m_{t} f(\lambda) d \lambda-\hat{\lambda}_{t}^{m} f\left(\hat{\lambda}^{m}\right) m\left(t, \hat{\lambda}^{m}\right)\right) / \hat{X}_{t} .
$$

Since $m_{t}>0$ and $\hat{\lambda}_{t}^{m} f\left(\hat{\lambda}^{m}\right) m\left(t, \hat{\lambda}^{m}\right) \geq 0$ (from (17)), the emission tax can be less than, equal to, or even greater than marginal damage. In the limiting case that administrative costs are all fixed costs (so that $m_{t}=0$ ), the optimal emissions tax is strictly greater than marginal damage.

Polinsky and Shavell (1982) have already noted these results, but they assumed exogenous distributions of administrative costs. Therefore, their results concerning optimal emissions taxes are relevant to situations in which the distribution of administrative costs does not affect social welfare. Our remaining results consider situations in which the distribution of these costs does matter. In all cases the emissions tax should not exceed marginal damage.

Our next proposition indicates conditions under which it is optimal for regulated firms to shoulder all administrative costs. These are not the only circumstances under which it is optimal to do so, but they do highlight the impacts of the distribution of administrative costs on industry size, the effect of the difference in the costs of public and private funds, and how the distribution of administrative costs affects equilibrium aggregate emissions.

Proposition 2: The optimal policy involves $\alpha=0$ and $t<d$ under the following circumstances:

[1] $\lambda_{\alpha}^{m} \approx 0$ and $\mu>1$;

[2] $\lambda_{\alpha}^{m}<0, \mu \geq 1$, and $\hat{X}_{\alpha} \geq 0$ for all $\alpha \in[0,1]$.

Proposition 2 indicates that it is optimal to have firms bear all administrative costs if the distribution of these costs has a negligible impact on the size of the industry, but public funds are more costly than private funds (condition [1] of the Proposition). When there is an industry size 
effect, the key is how the distribution of costs affects equilibrium aggregate emissions. If aggregate emissions do not decrease as the public takes on more of the administrative costs, then it is efficient to push all these costs onto firms. Imagine, for a given emissions tax, that having the public shoulder more of the administrative costs increases the size of the industry. This adds to administrative costs because there are more firms to control. Moreover, ignoring the effect on the output market for a moment, more firms under a fixed emissions tax leads to higher aggregate emissions. Thus, making firms bear all administrative costs reduces these costs by reducing the number of regulated firms and also reduces aggregate emissions. Of course, both of these effects might be mitigated by the workings of the output market, but the conclusion still holds as long as equilibrium aggregate emissions are non-decreasing in $\alpha$.

As noted at the end of section 2.3, $\hat{X}_{\alpha}>0$ if the regulated firms face a perfectly elastic demand curve. Thus, condition [2] of Proposition 2 is particularly relevant for domestic control of firms that are a small part of an international market. When the size of such an industry depends on the distribution of the administrative costs of an emissions tax, it is optimal to have the industry bear all of these costs

In all cases in which it is optimal to have firms bear all administrative costs, the optimal tax should not exceed marginal damage. To see why this is true, set $\alpha=0$ in (27) to obtain

$$
W_{t}(t, \alpha)=(t-d) \hat{X}_{t}-\int_{\hat{\lambda}^{m}}^{\bar{\lambda}} m_{t} f(\lambda) d \lambda=0
$$

$(t-d) \hat{X}_{t}$ indicates how an increase in the emissions tax affects the difference between aggregate abatement costs and damage. First best, of course, requires $t=d$, but the increase in aggregate administrative costs from a higher tax suggests that a lower level of emissions control is optimal; hence, $t<d$. 
The first part of our next proposition is a direct corollary of Propositions 1 and 2

Proposition 3: The optimal policy calls for $\alpha \in(0,1]$ only if $\hat{X}_{\alpha}<0$. If the optimal policy involves $\alpha \in(0,1]$, then $t<d$.

The only way that it would be optimal for the public to bear part of the administrative costs associated with an emissions tax is if equilibrium aggregate emissions are strictly decreasing in the public's share of administrative costs. In this case, having the public bear some administrative costs provides extra emissions control beyond what is provided by the emissions tax. As in all other cases, the emissions tax should not exceed marginal damage.

An example of when it is optimal for the public to bear some administrative costs is when there is no difference between the costs of public and private funds $(\mu=1)$ and $\hat{X}_{\alpha}<0$. In this case, if all the administrative costs were placed on the firms, then the first order conditions (27) and (28) would be:

$$
\begin{aligned}
& W_{t}(t, \alpha)=(t-d) \hat{X}_{t}-\int_{\hat{\lambda}^{m}}^{\bar{\lambda}} m_{t} f(\lambda) d \lambda=0 ; \\
& W_{\alpha}(t, \alpha)=(t-d) \hat{X}_{\alpha} \leq 0 .
\end{aligned}
$$

But these cannot characterize an optimal policy, because $W_{t}(t, \alpha)=0$ requires $t<d$, while $W_{\alpha}(t, \alpha) \leq 0$ requires $t>d$. Therefore, the optimal policy has the public taking on a part of the administrative costs when $\mu=1$ and $\hat{X}_{\alpha}<0$.

It is important to emphasize that $\hat{X}_{\alpha}<0$ is only a necessary condition for it to be efficient that public bear some administrative costs - it is easy to come up with circumstances 
under which equilibrium emissions are decreasing in the public's share of these costs, but it is optimal for the public to bear none of them. All of these situations involve the costs of public funds being higher than the cost of private funds.

\section{Conclusion}

We have examined theoretically the efficient distribution of the administrative costs associated with an emissions tax between regulated firms and the general public via government regulators. Our results suggest two ways in which the distribution of administrative costs matters. Higher costs of public funds over private funds favor placing more of this cost burden on polluters. In addition, the distribution of these costs can affect the size of a regulated industry. If public funds and private funds are equally costly, and if the distribution of administrative costs has a negligible effect on industry size, then the distribution of these costs does not have welfare consequences.

However, if the number of regulated firms is decreasing in their administrative cost burden, then the key to determining the optimal distribution of administrative costs is how the change in industry size affects equilibrium aggregate emissions. A sufficient condition for it to be optimal for firms to bear all administrative costs is that their equilibrium emissions do not increase as more of these costs are imposed on them. A necessary, but not sufficient, condition for it to be optimal that the public bear some part of administrative costs is that firms' emissions increase with their share of these costs. While our results suggest that the determination of the optimal distribution of administrative costs rests on empirical results that may vary from setting to setting, when the distribution actually matters it appears that the circumstances under which it 
is optimal for the public to bear part of administrative costs are more limited than those that require placing all of this burden on polluters.

Of course, the political opposition to emissions taxes is generally intense, and making firms bear all the costs of administering a tax can only enhance that opposition. Policy designers may relieve some of the administrative cost burden of regulated firms to make an emission tax more palatable. This may come at a cost if it is optimal to have the firms bear these costs, but it may be an efficiency-enhancing move if the alternative is a policy that is less efficient than an emissions tax. In cases in which the distribution of administrative costs is not chosen optimally, it is important to remember that the chosen distribution does affect the optimal emissions tax.

A more politically feasible option may be to introduce an emissions trading program with freely-allocated emissions permits. We doubt that our results about distributing the administrative costs of an emissions tax can be applied directly to emissions trading. The analysis is likely to be more complex because the distribution of administrative costs can affect two markets — the output and permit markets — simultaneously. Examining the optimal distribution of enforcement costs under emission trading is an important area for future research. 


\section{Appendix}

\section{Derivation of first-order conditions (27) and (28)}

From (26) the first order condition for the optimal choice of a strictly positive emissions tax is

$$
\begin{aligned}
W_{t}(t, \alpha) & =p(\hat{Q})\left(\int_{\hat{\lambda}^{m}}^{\bar{\lambda}} \hat{q}_{t} f(\lambda) d \lambda-\hat{\lambda}_{t}^{m} \hat{q}^{m} f\left(\hat{\lambda}^{m}\right)\right) \\
& -\left(\int_{\hat{\lambda}^{m}}^{\bar{\lambda}}\left(c_{q} \hat{q}_{t}+c_{x} \hat{x}_{t}\right) f(\lambda) d \lambda-\hat{\lambda}_{t}^{m} f\left(\hat{\lambda}^{m}\right) c\left(\hat{q}^{m}, \hat{x}^{m}, \hat{\lambda}^{m}\right)\right) \\
& -d\left(\int_{\hat{\lambda}^{m}}^{\bar{\lambda}} \hat{x}_{t} f(\lambda) d \lambda-\hat{\lambda}_{t}^{m} f\left(\hat{\lambda}^{m}\right) \hat{x}^{m}\right) \\
& -(1+\alpha(\mu-1))\left(\int_{\hat{\lambda}^{m}}^{\bar{\lambda}} m_{t} f(\lambda) d \lambda-\hat{\lambda}_{t}^{m} f\left(\hat{\lambda}^{m}\right) m\left(t, \hat{\lambda}^{m}\right)\right)=0 .
\end{aligned}
$$

From (2) substitute $p(\hat{Q})=c_{q}$ and $t=-c_{x}$ into (A.1) and collect terms to obtain

$$
\begin{aligned}
W_{t}(t, \alpha) & =(t-d) \int_{\hat{\lambda}^{m}}^{\bar{\lambda}} \hat{x}_{t} f(\lambda) d \lambda-(1+\alpha(\mu-1)) \int_{\hat{\lambda}^{m}}^{\bar{\lambda}} m_{t} f(\lambda) d \lambda \\
& -\hat{\lambda}_{t}^{m} f\left(\hat{\lambda}^{m}\right)\left[p(\hat{Q}) \hat{q}^{m}-c\left(\hat{q}^{m}, \hat{x}^{m}, \hat{\lambda}^{m}\right)-d \hat{x}^{m}-(1+\alpha(\mu-1)) m\left(t, \hat{\lambda}^{m}\right)\right] .
\end{aligned}
$$

Substitute equilibrium values of the cut-off firm into (5) to obtain

$$
\pi\left(t, \alpha, \hat{p}, \hat{\lambda}^{m}\right)=p(\hat{Q}) \hat{q}^{m}-c\left(\hat{q}^{m}, \hat{x}^{m}, \hat{\lambda}^{m}\right)-t \hat{x}^{m}-(1-\alpha) m\left(t, \hat{\lambda}^{m}\right) \equiv 0 .
$$

Subtract $d \hat{x}^{m}$ and $\alpha \mu m\left(t, \hat{\lambda}^{m}\right)$ from both sides of (A.3) and rearrange terms to obtain

$$
\begin{gathered}
p(\hat{Q}) \hat{q}^{m}-c\left(\hat{q}^{m}, \hat{x}^{m}, \hat{\lambda}^{m}\right)-d \hat{x}^{m}-(1+\alpha(\mu-1)) m\left(t, \hat{\lambda}^{m}\right) \\
=(t-d) d \hat{x}^{m}-\alpha \mu m\left(t, \hat{\lambda}^{m}\right) .
\end{gathered}
$$

Substitute (A.4) into (A.2) and rearrange terms to obtain

$$
\begin{aligned}
W_{t}(t, \alpha) & =(t-d)\left(\int_{\hat{\lambda}^{m}}^{\bar{\lambda}} \hat{x}_{t} f(\lambda) d \lambda-\hat{\lambda}_{t}^{m} f\left(\hat{\lambda}^{m}\right) \hat{x}^{m}\right)-(1+\alpha(\mu-1)) \int_{\hat{\lambda}^{m}}^{\bar{\lambda}} m_{t} f(\lambda) d \lambda \\
& +\hat{\lambda}_{t}^{m} f\left(\hat{\lambda}^{m}\right) \alpha \mu m\left(t, \hat{\lambda}^{m}\right)=0 .
\end{aligned}
$$


Substitute $\hat{X}_{t}=\int_{\hat{\lambda}^{m}}^{\bar{\lambda}} \hat{x}_{t} f(\lambda) d \lambda-\hat{\lambda}_{t}^{m} f\left(\hat{\lambda}^{m}\right) \hat{x}^{m}<0$ into (A.5) to obtain (27).

Now turn to the first-order condition for $\alpha$, which from (26) is

$$
\begin{aligned}
W_{\alpha}(t, \alpha) & =p(\hat{Q})\left(\int_{\hat{\lambda}^{m}}^{\bar{\lambda}} \hat{q}_{\alpha} f(\lambda) d \lambda-\hat{\lambda}_{\alpha}^{m} \hat{q}^{m} f\left(\hat{\lambda}^{m}\right)\right) \\
& -\left(\int_{\hat{\lambda}^{m}}^{\bar{\lambda}}\left(c_{q} \hat{q}_{\alpha}+c_{x} \hat{x}_{\alpha}\right) f(\lambda) d \lambda-\hat{\lambda}_{\alpha}^{m} f\left(\hat{\lambda}^{m}\right) c\left(\hat{q}^{m}, \hat{x}^{m}, \hat{\lambda}^{m}\right)\right) \\
& -d\left(\int_{\hat{\lambda}^{m}}^{\bar{\lambda}} \hat{x}_{\alpha} f(\lambda) d \lambda-\hat{\lambda}_{\alpha}^{m} f\left(\hat{\lambda}^{m}\right) \hat{x}^{m}\right) \\
& -(\mu-1) \int_{\hat{\lambda}^{m}}^{\bar{\lambda}} m(t, \lambda) f(\lambda) d \lambda \\
& +(1+\alpha(\mu-1)) \hat{\lambda}_{\alpha}^{m} f\left(\hat{\lambda}^{m}\right) m\left(t, \hat{\lambda}^{m}\right)\left\{\begin{array}{l}
\leq 0, \text { if }<0, \text { then } \alpha=0 \\
\geq 0, \text { if }>0, \text { then } \alpha=1 .
\end{array}\right.
\end{aligned}
$$

Substitute $p(\hat{Q})=c_{q}$ and $t=-c_{x}$ into (A.6) and collect terms to obtain

$$
\begin{aligned}
W_{\alpha}(t, \alpha) & =(t-d) \int_{\hat{\lambda}^{m}}^{\bar{\lambda}} \hat{x}_{\alpha} f(\lambda) d \lambda-\hat{\lambda}_{\alpha}^{m} f\left(\hat{\lambda}^{m}\right)\left[p(\hat{Q}) \hat{q}^{m}-c\left(\hat{q}^{m}, \hat{x}^{m}, \hat{\lambda}^{m}\right)-d \hat{x}^{m}\right. \\
& -\left(1+\alpha(\mu-1) m\left(t, \hat{\lambda}^{m}\right)\right]-(\mu-1) \int_{\hat{\lambda}^{m}}^{\bar{\lambda}} m(t, \lambda) f(\lambda) d \lambda\left\{\begin{array}{l}
\leq 0, \text { if }<0, \text { then } \alpha=0 \\
\geq 0, \text { if }>0, \text { then } \alpha=1 .
\end{array}\right.
\end{aligned}
$$

Substitute (A.4) into (A.7) and collect terms to obtain

$$
\begin{aligned}
W_{\alpha}(t, \alpha)= & (t-d)\left(\int_{\hat{\lambda}^{m}}^{\bar{\lambda}} \hat{x}_{\alpha} f(\lambda) d \lambda-\hat{\lambda}_{\alpha}^{m} f\left(\hat{\lambda}^{m}\right) \hat{x}^{m}\right)+\hat{\lambda}_{\alpha}^{m} f\left(\hat{\lambda}^{m}\right) \alpha \mu m\left(t, \hat{\lambda}^{m}\right) \\
& -(\mu-1) \int_{\hat{\lambda}^{m}}^{\bar{\lambda}} m(t, \lambda) f(\lambda) d \lambda\left\{\begin{array}{l}
\leq 0, \text { if }<0, \text { then } \alpha=0 \\
\geq 0, \text { if }>0, \text { then } \alpha=1 .
\end{array}\right.
\end{aligned}
$$

Substitute $\hat{X}_{\alpha}=\int_{\hat{\lambda}^{m}}^{\bar{\lambda}} \hat{x}_{\alpha} f(\lambda) d \lambda-\hat{\lambda}_{\alpha}^{m} f\left(\hat{\lambda}^{m}\right) \hat{x}^{m}$ into (A.8) to obtain (28). 
Proof of Proposition 1: Recall that $\lambda_{\alpha}^{m} \approx 0$ implies $\hat{p}_{\alpha} \approx 0$. In turn, from (18) and (19), $\hat{x}_{\alpha} \approx \hat{\lambda}_{\alpha}^{m} \approx 0$, which further imply $\hat{X}_{\alpha} \approx 0$. Thus, if $\lambda_{\alpha}^{m}$ is negligible then the first two terms in (A.8) are independent of $\alpha$. If $\mu=1$ as well, then $W_{\alpha}(t, \alpha)=0$ independently of $\alpha$. Therefore, social welfare is unaffected by the distribution of administrative costs when $\lambda_{\alpha}^{m} \approx 0$ and $\mu=1$.

Proof of Proposition 2: Under conditions [1] of the Proposition, since $\lambda_{\alpha}^{m} \approx 0$ implies $\hat{\lambda}_{\alpha}^{m} \approx 0$ and $\hat{X}_{\alpha} \approx 0$, (28) becomes

$$
W_{\alpha}(t, \alpha) \approx-(\mu-1) \int_{\hat{\lambda}^{m}}^{\bar{\lambda}} m_{t} f(\lambda) d \lambda<0
$$

the sign of which follows from $\mu>1$ and $m_{t}>0$. Of course, $W_{\alpha}(t, \alpha)<0$ means the optimal $\alpha$ is zero. Then, (27) becomes

$$
W_{t}(t, \alpha)=(t-d) \hat{X}_{t}-\int_{\hat{\lambda}^{m}}^{\bar{\lambda}} m_{t} f(\lambda) d \lambda=0 .
$$

Since $\hat{X}_{t}<0$ and $m_{t}>0, t<d$ is required for the first order condition to hold.

Under the conditions [2] of the Proposition, note first that

$$
\hat{X}_{\alpha}=\int_{\hat{\lambda}^{m}}^{\bar{\lambda}} \hat{x}_{\alpha} f(\lambda) d \lambda-\hat{\lambda}_{\alpha}^{m} f\left(\hat{\lambda}^{m}\right) \hat{x}^{m} \geq 0
$$

requires $\hat{x}_{\alpha}<0$ because $\lambda_{\alpha}^{m}<0$ implies $\hat{\lambda}_{\alpha}^{m}<0$ from (20). Toward a contradiction of the Proposition suppose that the optimal policy involves $\alpha \in(0,1]$. From (28) this requires $W_{\alpha}(t, \alpha) \geq 0$. Moreover, since 


$$
-(\mu-1) \int_{\hat{\lambda}^{m}}^{\bar{\lambda}} m_{t} f(\lambda) d \lambda+\hat{\lambda}_{\alpha}^{m} f\left(\hat{\lambda}^{m}\right) \alpha \mu m\left(t, \hat{\lambda}^{m}\right)<0,
$$

because $\mu \geq 1$ and $\hat{\lambda}_{\alpha}^{m}<0, W_{\alpha}(t, \alpha) \geq 0$ implies $\hat{X}_{\alpha} \neq 0$. Since $\hat{X}_{\alpha} \geq 0$ by assumption, we must have $\hat{X}_{\alpha}>0$. Given (A.12), $\hat{X}_{\alpha}>0$ implies that $t>d$ is required for $W_{\alpha}(t, \alpha) \geq 0$.

Now substitute (A.11) in for $\hat{X}_{\alpha}$ in $W_{\alpha}(t, \alpha) \geq 0$ and rearrange terms to obtain:

$$
\begin{aligned}
W_{\alpha}(t, \alpha) & =(t-d) \int_{\hat{\lambda}^{m}}^{\bar{\lambda}} \hat{x}_{\alpha} f(\lambda) d \lambda-\hat{\lambda}_{\alpha}^{m} f\left(\hat{\lambda}^{m}\right)\left[(t-d) \hat{x}^{m}-\alpha \mu m\left(t, \hat{\lambda}^{m}\right)\right] \\
& -(\mu-1) \int_{\hat{\lambda}^{m}}^{\bar{\lambda}} m_{t} f(\lambda) d \lambda \geq 0 .
\end{aligned}
$$

Since $t>d$ and $\hat{x}_{\alpha}<0,(t-d) \int_{\hat{\lambda}^{m}}^{\bar{\lambda}} \hat{x}_{\alpha} f(\lambda) d \lambda<0$. This along with $-(\mu-1) \int_{\hat{\lambda}^{m}}^{\bar{\lambda}} m_{t} f(\lambda) d \lambda \leq 0$ and $\hat{\lambda}_{\alpha}^{m}<0$ imply that

$$
(t-d) \hat{x}^{m}-\alpha \mu m\left(t, \hat{\lambda}^{m}\right)>0
$$

is required for (A.13). Next, substitute

$$
\hat{X}_{t}=\int_{\hat{\lambda}^{m}}^{\bar{\lambda}} \hat{x}_{t} f(\lambda) d \lambda-\hat{\lambda}_{t}^{m} f\left(\hat{\lambda}^{m}\right) \hat{x}^{m}<0
$$

into (27) and rearrange the result to obtain

$$
(t-d) \int_{\hat{\lambda}^{m}}^{\bar{\lambda}} \hat{x}_{t} f(\lambda) d \lambda=(1+\alpha(\mu-1)) \int_{\hat{\lambda}^{m}}^{\bar{\lambda}} m_{t} f(\lambda) d \lambda+\hat{\lambda}_{t}^{m} f\left(\hat{\lambda}^{m}\right)\left[(t-d) \hat{x}^{m}-\alpha \mu m\left(t, \hat{\lambda}^{m}\right)\right]
$$

The right side of (A.15) is positive, because $(1+\alpha(\mu-1)) \int_{\hat{\lambda}^{m}}^{\bar{\lambda}} m_{t} f(\lambda) d \lambda>0, \hat{\lambda}_{t}^{m} f\left(\hat{\lambda}^{m}\right) \geq 0$ (from (17)), and $(t-d) \hat{x}^{m}-\alpha \mu m\left(t, \hat{\lambda}^{m}\right) \geq 0$ from (A.14), which is required to satisfy $W_{\alpha}(t, \alpha) \geq 0$.

However, to make the left side positive we must have $t<d$ because $\int_{\lambda^{m}}^{\bar{\lambda}} \hat{x}_{t} f(\lambda) d \lambda<0$. Thus, we have our contradiction, because $\alpha \in(0,1]$ requires $t>d$ to satisfy $W_{\alpha}(t, \alpha) \geq 0$, but it requires 
$t<d$ to satisfy $W_{t}(t, \alpha)=0$. Since $\alpha \notin(0,1]$ under conditions [2] of Proposition 2, an optimal policy in this situation must involve $\alpha=0$. To show the optimal policy under these conditions also requires $t<d$, substitute $\alpha=0$ into (A.15) to obtain

$$
W_{t}(t, \alpha)=(t-d) \hat{X}_{t}-\int_{\hat{\lambda}^{m}}^{\bar{\lambda}} m_{t} f(\lambda) d \lambda=0
$$

the satisfaction of which requires $t<d$ because $\hat{X}_{t}<0$ and $-\int_{\hat{\lambda}^{m}}^{\bar{\lambda}} m_{t} f(\lambda) d \lambda<0$

Proof of Proposition 3: Toward a contradiction of the first part of the Proposition, suppose that $\alpha \in(0,1]$ when $\hat{X}_{\alpha} \geq 0$. It is possible that $\hat{X}_{\alpha} \approx 0$, because $\lambda_{\alpha}^{m} \approx 0$. However, when this occurs either the optimal policy is independent of $\alpha$ (Proposition 1) or the optimal policy involves $\alpha=0$ (Proposition 2). When $\lambda_{\alpha}^{m}<0$ and $\hat{X}_{\alpha} \geq 0$, the optimal policy involves $\alpha=0$ (Proposition 2). Thus, it is only possible that the optimal policy has $\alpha \in(0,1]$ when $\hat{X}_{\alpha}<0$.

We now prove the second part of the Proposition. Assume that the optimal policy involves $\alpha \in(0,1]$. This requires $\hat{X}_{\alpha}<0$ and

$$
W_{\alpha}(t, \alpha)=(t-d) \hat{X}_{\alpha}-(\mu-1) \int_{\hat{\lambda}^{m}}^{\bar{\lambda}} m_{t} f(\lambda) d \lambda+\hat{\lambda}_{\alpha}^{m} f\left(\hat{\lambda}^{m}\right) \alpha \mu m\left(t, \hat{\lambda}^{m}\right) \geq 0
$$

Given $-(\mu-1) \int_{\hat{\lambda}^{m}}^{\bar{\lambda}} m_{t} f(\lambda) d \lambda \leq 0, \hat{\lambda}_{\alpha}^{m} f\left(\hat{\lambda}^{m}\right) \alpha \mu m\left(t, \hat{\lambda}^{m}\right)<0$, and $\hat{X}_{\alpha}<0$, satisfaction of (A.16) requires $t<d$ 


\section{References}

Brock, William A. and David S. Evans. 1985. "The economics of regulatory tiering." RAND Journal of Economics 16(3), 398-409.

Cremer, Helmuth and Firouz Gahvari. 2002. "Imperfect observability of emissions and secondbest emission and output taxes." Journal of Public Economics 85(3), 385-407.

Harford, Jon. 1987. "Self-reporting of pollution and the firm's behavior under imperfectly enforceable regulations." Journal of Environmental Economics and Management 14(3), 293303.

Harford, Jon. 1978. "Firm behavior under imperfectly enforceable pollution standards and taxes." Journal of Environmental Economics and Management 5(1), $26-43$.

Kampas, Athanasios and Ben White. 2004. "Administrative costs and instrument choice for stochastic non-point source pollutants." Environmental and Resource Economics 27, 109-133.

Macho-Stadler, Ines and David Perez-Castrillo. 2006. "Optimal enforcement policy and firm's emissions and compliance with environmental taxes." Journal of Environmental Economics and Management 51(1), 110-131.

Montero, Juan-Pablo. 2002. "Prices versus quantities with incomplete enforcement." Journal of Public Economics 85(3), 435-454.

Polinsky, A. Mitchell and Steven Shavell. 1982. "Pigouvian taxation with administrative costs." Journal of Public Economics 19, 385-394

Sandmo, Agnar. 2002. "Efficient environmental policy with imperfect compliance." Environmental and Resource Economics 23(1), 85-103.

Schmutzler, Armin and Lawrence H. Goulder. 1997. "The choice between emission taxes and output taxes under imperfect monitoring." Journal of Environmental Economics and Management 32, 51-64.

Schrank, William E.; Ragnar Arnason, and Rognvaldur Hannesson. 2003. The Cost of Fisheries Management. Ashgate Publishing.

Smulders, Sjak and Herman R. J. Vollebergh. 2001. "Green taxes and administrative costs: the case of carbon taxation." In Behavioral and Distributional Effects of Environmental Policy, Carlo Carraro and Gilbert E. Metcalf (Eds.), University of Chicago Press.

Stranlund, John K.; Carlos A. Chavez, and Mauricio G. Villena. 2009. "The optimal pricing of pollution when enforcement is costly." Journal of Environmental Economics and Management 58(2), 183-191.

Tietenberg, Tom. 2003. "The tradable-permits approach to protecting the commons: Lessons for climate change." Oxford Review of Economic Policy 19(3), 400-419. 